\title{
Quasisymmetric nonparametrization and spaces associated with the Whitehead continuum
}

\author{
JUHA HEINONEN \\ JANG-MEI WU
}

\begin{abstract}
The decomposition space $\mathbf{R}^{3} / W h$ associated with the Whitehead continuum Wh is not a manifold, but the product $\left(\mathbf{R}^{3} / W h\right) \times \mathbf{R}^{m}$ is homeomorphic to $\mathbf{R}^{3+m}$ for any $m \geq 1$ (known since the 1960's). We study the quasisymmetric structure on $\left(\mathbf{R}^{3} / W h\right) \times \mathbf{R}^{m}$ and show that the space $\left(\mathbf{R}^{3} / W h\right) \times \mathbf{R}^{m}$ may be equipped with a metric resembling $\mathbf{R}^{3+m}$ geometrically and measure theoretically-it is linearly locally contractible and Ahlfors $(3+m)$-regular-nevertheless the resulting space does not admit a quasisymmetric parametrization by $\mathbf{R}^{3+m}$.
\end{abstract}

$30 \mathrm{C} 65 ; 57 \mathrm{~N} 16,57 \mathrm{~N} 45$

In memory of Juha Heinonen, an extraordinary mathematician and friend, who passed away after the mathematical work in this paper was completed. $-J-M W$

\section{Introduction}

To understand the underlying structure of a metric space, one seeks a parametrization of a special type. The classical Riemann mapping theorem is an example. The problem of characterizing metric $n$-spheres that are bi-Lipschitz equivalent to the standard sphere $\mathbf{S}^{n}$, or quasisymmetrically equivalent to $\mathbf{S}^{n}$, has received considerable attention in recent years. Despite a large number of results (for example by Bonk and Kleiner [6], David and Semmes [10], Heinonen and Koskela [18], Laakso [22] and Semmes [29; $31 ; 32])$, the question is still far from being understood.

There exist finite 5-dimensional polyhedra that are homeomorphic to the standard sphere $\mathbf{S}^{5}$ but not bi-Lipschitz equivalent to $\mathbf{S}^{5}$-an observation of Siebenmann and Sullivan [34] based on deep work of Cannon [7] and Edwards [12; 11], which asserts that the double suspension $\Sigma^{2} H^{3}$ of a 3-dimensional homology sphere $H^{3}$ with a nontrivial fundamental group is homeomorphic to the standard sphere $\mathbf{S}^{5}$. The double suspension $\Sigma^{2} H^{3}$ can be considered as the join $\mathbf{S}^{1} * H^{3}$, where $H^{3}$ is triangulated and $\Sigma^{2} H^{3}$ is equipped with a natural barycenter metric. The complement of the suspension circle $\mathbf{S}^{1}$ in $\Sigma^{2} H^{3}$ is not simply connected; therefore every homeomorphism $f$ 
from $\Sigma^{2} H^{3}$ onto $\mathbf{S}^{5}$ must map $\mathbf{S}^{1}$ onto a curve $f\left(\mathbf{S}^{1}\right)$ whose complement in $\mathbf{S}^{5}$ is not simply connected. Consequently $f\left(\mathbf{S}^{1}\right)$ has positive 3 -dimensional Hausdorff measure. Hence $f$ can not be Hölder continuous of order greater than $1 / 3$ and is not bi-Lipschitz. It was then asked by Siebenmann and Sullivan [34] whether there exists a quasisymmetric homeomorphism between $\Sigma^{2} H^{3}$ and $\mathbf{S}^{5}$, a question which seems inaccessible at the moment.

We study a problem in a similar spirit and, in some sense, of one dimension lower. Let $W h$ denote a Whitehead continuum in $\mathbf{R}^{3}$ (see Whitehead [39]) and $\mathbf{R}^{3} / W h$ be the decomposition space associated with $W h$ obtained by collapsing $W h$ to a point $q$ while leaving all other points unchanged. The resulting space $\mathbf{R}^{3} / W h$ endowed with the quotient topology is not a manifold at $q$, therefore is not homeomorphic to $\mathbf{R}^{3}$; however the product space $\left(\mathbf{R}^{3} / W h\right) \times \mathbf{R}^{1}$ is homeomorphic to $\mathbf{R}^{4}$ (see Bing [4; 5]). The complement of $\{q\} \times \mathbf{R}^{1}$ in $\left(\mathbf{R}^{3} / W h\right) \times \mathbf{R}^{1}$ is not simply connected at $\{q\} \times \mathbf{R}^{1}$. Therefore any homeomorphism $f$ from $\left(\mathbf{R}^{3} / W h\right) \times \mathbf{R}^{1}$ onto $\mathbf{R}^{4}$ maps $\{q\} \times \mathbf{R}^{1}$ to an infinite arc, which is wild in $\mathbf{R}^{4}$ and necessarily has positive 2-dimensional Hausdorff measure. Hence $f$ can not be Hölder continuous of order greater than $1 / 2$.

Semmes [31, page 206] constructed a manifold $\widetilde{M}$ modeled on $\mathbf{R}^{3} / W h$ and showed that $\widetilde{M}$ can be realized as a linearly locally contractible (resembling $\mathbf{R}^{3}$ geometrically) and Ahlfors 3-regular (resembling $\mathbf{R}^{3}$ measure theoretically) subset of $\mathbf{R}^{4}$; on the other hand $\widetilde{M}$ is not quasisymmetrically equivalent to $\mathbf{R}^{3}$. Heinonen and Semmes [20] asked the following question: When $\left(\mathbf{R}^{3} / W h\right) \times \mathbf{R}^{m}$ is equipped with a linearly locally contractible and Ahlfors $(3+m)$-regular metric, does $\left(\mathbf{R}^{3} / W h\right) \times \mathbf{R}^{m}$ then admit a quasisymmetric parametrization by $\mathbf{R}^{3+m}$ for some $m \geq 1$ ? We answer this question in the negative using essentially a product of the Semmes metric with the Euclidean metric.

1.1 Theorem For any $m \geq 1$, the metric space $\left(\mathbf{R}^{3} / W h\right) \times \mathbf{R}^{m}$, though homeomorphic to $\mathbf{R}^{3+m}$, can be equipped with a metric that is linearly locally contractible and Ahlfors $(3+m)$-regular so that the resulting space does not admit a quasisymmetric parametrization by $\mathbf{R}^{3+m}$.

The deeper reason for the nonexistence of the quasisymmetric parametrization in Theorem 1.1 is the fact that the meridians of the solid tori used in construction of the Whitehead continuum $W h$ are trivial in homology of the complement of $W h$, but nontrivial in homotopy.

We now proceed to give the formal definitions. 
A homeomorphism $f: X \rightarrow Y$ between two metric spaces is said to be bi-Lipschitz if there is a constant $C \geq 1$ so that

$$
C^{-1}|x-y| \leq|f(x)-f(y)| \leq C|x-y|
$$

for all $x, y$ in $X$. A homeomorphism $f: X \rightarrow Y$ between two metric spaces is said to be quasisymmetric if there is a homeomorphism $\eta:[0, \infty) \rightarrow[0, \infty)$ so that

$$
|x-a| \leq t|x-b| \text { implies }|f(x)-f(a)| \leq \eta(t)|f(x)-f(b)|
$$

for all triples of points $x, a, b$ in $X$ and for all $t>0$ (see Tukia and Väisälä [35]). Quasisymmetric maps distort relative distance by a finite amount and distort distance roughly the same in all directions. They are, in some sense, appropriate generalizations of conformal mappings for metric spaces.

A metric space is said to be linearly locally contractible if there is a constant $C \geq 1$ so that each metric ball of radius $0<r<C^{-1}$ in the space can be contracted to a point inside the ball of same center with radius $C r$. Linear local contractibility is a geometric condition; it is necessarily satisfied if a metric space is quasisymmetrically equivalent to $\mathbf{R}^{n}$.

A metric space $X$ is said to be Ahlfors $Q$-regular if there is a Borel measure $\mu$ such that

$$
C^{-1} r^{Q} \leq \mu\left(B_{r}\right) \leq C r^{Q}
$$

for some constants $Q>0$ and $C \geq 1$, and for all closed balls $B_{r}$ of radius $0<$ $r \leq \operatorname{diam} X$. In this case, $X$ has Hausdorff dimension $Q$ and (1.2) holds when $\mu$ is replaced with the Hausdorff $Q$-measure of $X$ (possibly with a different constant $C$ ). When $Q$ is an integer, the space $X$ resembles $\mathbf{R}^{Q}$ measure theoretically but may be totally disconnected. A metric space may be quasisymmetrically equivalent to $\mathbf{S} Q$ but not $Q$-regular; the von Koch snowflake curve is an example.

A theorem of Semmes [30] states that if a linearly locally contractible topological $Q$-manifold is Ahlfors $Q$-regular, then it satisfies a $(1,1)$-Poincaré inequality, hence it has nice analytical properties.

Tukia and Väisälä [35] have shown that a metric doubling space $(X, d)$ homeomorphic to $\mathbf{S}^{1}$ is quasisymmetrically equivalent to $\mathbf{S}^{1}$ if and only if $X$ satisfies the Ahlfors 2-point condition [35].

Bonk and Kleiner [6] have given a necessary and sufficient condition for a doubling metric space to be quasisymmetrically equivalent to $\mathbf{S}^{2}$. With this they proved the following theorem: A linearly locally contractible metric 2-sphere is quasisymmetrically equivalent to $\mathbf{S}^{2}$ if it is Ahlfors 2-regular. 
Semmes [31] showed that the theorem of Bonk and Kleiner fails in dimension 3 for a geometrical realization of the decomposition space associated with the Bing double in $\mathbf{R}^{3}$, and for the manifold $\widetilde{M}$ mentioned earlier. Theorem 1.1 shows that these natural conditions for quasisymmetric parametrization are also insufficient in dimension 4 and higher.

Acknowledgements Detailed comments of Mario Bonk, Pekka Pankka and the referee on the original manuscript are greatly appreciated. They have clarified the mathematics and improved the exposition.

JH was supported in part by NSF grants DMS-0353549 and DMS-0652915. J-MW was supported in part by NSF grants DMS-0400810 and DMS-0653088 and by the Frederick W and Lois B Gehring Professorship at the University of Michigan where the research was initiated. J-MW thanks the Department of Mathematics at Michigan for the hospitality.

\section{Whitehead continuum}

In 1935, JHC Whitehead [39] discovered a contractible noncompact 3-manifold in $\mathbf{S}^{3}$ that is not homeomorphic to $\mathbf{R}^{3}$. The complement of this manifold is now called a Whitehead continuum. Topological properties and pictures accompanying the construction of a Whitehead continuum can be found in Daverman [9], Kirby [21] and Semmes [33].

Given a set $E$ in $\left\{x_{1}=0, x_{2} \geq 0\right\} \subset \mathbf{R}^{3}$, with a slight abuse of notation, denote by $\mathbf{S}^{1} \times E$ the solid of revolution $\left\{\left(x_{1}, x_{2}, x_{3}\right):\left(0,\left(x_{1}^{2}+x_{2}^{2}\right)^{1 / 2}, x_{3}\right) \in E\right\}$ in $\mathbf{R}^{3}$. Let $B_{0}^{2}$ (resp. $\partial B_{0}^{2}$ ) denote the closed disk (resp. the circle) in $\left\{x_{1}=0\right\} \subset \mathbf{R}^{3}$ having center $(0,1,0)$ and radius $\frac{1}{2}$.

A simple closed curve $\alpha$ on $\mathbf{S}^{1} \times \partial B_{0}^{2}$ is a meridian (resp. a longitude) of the torus $\mathbf{S}^{1} \times B_{0}^{2}$ if there exists a homeomorphism from $\partial B_{0}^{2}\left(\right.$ resp. $\left.\mathbf{S}^{1} \times\left\{\left(0, \frac{1}{2}, 0\right)\right\}\right)$ onto $\alpha$ which is homotopic in $\mathbf{S}^{1} \times \partial B_{0}^{2}$ to the identity map on $\partial B_{0}^{2}\left(\right.$ resp. $\left.\mathbf{S}^{1} \times\left\{\left(0, \frac{1}{2}, 0\right)\right\}\right)$.

Let $T_{0}$ be a smooth compact solid torus embedded in $\mathbf{R}^{3}$, identified with $\mathbf{S}^{1} \times B_{0}^{2}$ for convenience. Let $T_{1}$ be a second compact solid torus smoothly embedded in the interior of $T_{0}$ and positioned in such a way that $T_{1}$ and any meridian of $T_{0}$ form a Whitehead link (see Kirby [21] and Rourke and Sanderson [27]). Note that $T_{1}$ is contractible in $T_{0}$, but the contraction of $T_{1}$ can not take place without crossing itself. In fact, $T_{1}$ is knotted in $T_{0}$ but is not knotted in $\mathbf{R}^{3}$. Let $\phi$ be a diffeomorphism defined in a neighborhood of $T_{0}$ that maps $T_{0}$ onto $T_{1}$. Define inductively for $k \geq 2$,

$$
T_{k}=\phi\left(T_{k-1}\right) \text {. }
$$


The intersection

$$
W h=\bigcap_{k=0}^{\infty} T_{k}
$$

is a Whitehead continuum. The continuum $W h$ is one-dimensional and is contractible inside each $T_{k}, k \geq 0$. Identify $\mathbf{S}^{3}$ with $\mathbf{R}^{3} \cup\{\infty\}$ in topology. The Whitehead manifold $\mathbf{S}^{\mathbf{3}} \backslash W h$ is simply connected and in fact contractible [21].

The Whitehead link is symmetric. For $k \geq 1$, there is a homeomorphism of $\mathbf{S}^{3}$ that interchanges a meridian of $T_{k}$ with a core of $T_{k+1}\left(\right.$ such as $\left.\phi^{(k+1)}\left(\mathbf{S}^{1} \times\{(0,1,0)\}\right)\right)$. However the homeomorphism reversing the link can not be confined to $T_{0}$ (see Kirby [21, page 83]). Therefore there are loops, namely the meridians of $T_{k}$, lying arbitrarily close to the Whitehead continuum $W h$ that can not be contracted in $\mathbf{S}^{3} \backslash W h$ to a point while staying near $W h$. In other words the Whitehead manifold $\mathbf{S}^{\mathbf{3}} \backslash W h$ is not simply connected at $W h$, so $\mathbf{S}^{3} \backslash W h$ is not homeomorphic to $\mathbf{R}^{3}$.

The decomposition space $\mathbf{R}^{3} / W h$ is defined by collapsing $W h$ to a single point, called $q$, while keeping all other points unchanged, then endowed with the quotient topology. The resulting space is not a manifold at the quotient point $q$ by the previous discussion, hence it is not homeomorphic to $\mathbf{R}^{3}$.

Taking the product with $\mathbf{R}^{m}$ ( $m \geq 1$ ) however destroys all nonmanifold points; as a consequence $\left(\mathbf{R}^{3} / W h\right) \times \mathbf{R}^{m}$ is homeomorphic to $\mathbf{R}^{3+m}$ (by Andrews and Rubin [3], also credited to Arnold Shapiro by Bing [4; 5]). See Daverman [9] and Kirby [21] for more discussions about products of decompositions with a line.

Let $\pi: \mathbf{R}^{3} \rightarrow \mathbf{R}^{3} / W h$ be the quotient map defined by $\pi(W h)=q$ and $\pi(x)=x$ if $x \notin$ $W h$; for simplicity denote by

$$
\widetilde{E}=\pi(E)
$$

the image of a set $E$ under $\pi$. Let

$$
\mathcal{L}=\{q\} \times \mathbf{R}^{m} .
$$

A curve $\gamma$ on $\partial \widetilde{T}_{k}$ is called a meridian (resp. a longitude) of $\widetilde{T}_{k}$ if $\pi^{-1} \gamma$ is a meridian (resp. a longitude) of $T_{k}$. The noncontractibility of the meridians of $T_{k}$ in the Whitehead manifold near $W h$ implies the following.

2.1 Proposition Meridians of $\widetilde{T}_{k}$ are not contractible in $\widetilde{T}_{0} \backslash\{q\}$, and meridians of $\widetilde{T}_{k} \times\{z\}$ are not contractible in $\left(\widetilde{T}_{0} \times \mathbf{R}^{m}\right) \backslash \mathcal{L}$, for any $m \geq 1, k \geq 1$ and $z \in \mathbf{R}^{m}$.

On the other hand, a meridian of $\widetilde{T}_{k}$ bounds a surface (a disk with a handle) in $\widetilde{T}_{k} \backslash\{q\}$. The difference of the roles of a meridian in homotopy and in homology is important in our proof. 


\section{The metric}

We define a metric on the decomposition space $\mathbf{R}^{3} /$ Wh by adapting that of Semmes in [31]. The metric to be defined has a self-similarity property and sizes of $\widetilde{T}_{k}$ decrease geometrically towards the point $q$. Semmes embedded $\mathbf{R}^{3} / W h$ in $\mathbf{R}^{4}$ which is more visual; we consider $\left(\mathbf{R}^{3} / W h\right) \backslash\{q\}$ as an abstract manifold on which the metric can be explicitly described.

Fix a number $c_{0} \in\left(0, \frac{1}{20}\right)$. Let $P_{0}=(0,1,0)$, and $A=\left\{x \in\{0\} \times \mathbf{R}^{2}: \frac{1}{2}-2 c_{0}<\right.$ $\left.\left|x-P_{0}\right| \leq \frac{1}{2}-c_{0}\right\}, A^{\prime}=\left\{x \in\{0\} \times \mathbf{R}^{2}: \frac{1}{2}-2 c_{0}<\left|x-P_{0}\right|<\frac{1}{2}+2 c_{0}\right\}$ and $A^{\prime \prime}=\left\{x \in\{0\} \times \mathbf{R}^{2}: \frac{1}{2}-3 c_{0}<\left|x-P_{0}\right|<\frac{1}{2}+3 c_{0}\right\}$ be three annuli in $\{0\} \times \mathbf{R}^{2}$. Let

$$
U_{0}=\mathbf{S}^{1} \times A, \quad V_{0}=\mathbf{S}^{1} \times A^{\prime} \quad \text { and } \quad W_{0}=\mathbf{S}^{1} \times A^{\prime \prime}
$$

be the solids obtained by revolving $A, A^{\prime}$ and $A^{\prime \prime}$ about the $x_{3}$-axis. Note that $V_{0}$ and $W_{0}$ are neighborhoods of $\partial T_{0}$ and that $U_{0} \subset T_{0} \cap V_{0} \subset V_{0} \subset W_{0}$. Recall that $\phi$ is a diffeomorphism defined in a neighborhood of $T_{0}$ that maps $T_{0}$ onto $T_{1}$. Assume that $c_{0}$ has been chosen small enough so that $W_{0}$ is contained in the neighborhood of $T_{0}$ on which $\phi$ is defined and that $\phi\left(T_{0} \cup W_{0}\right) \subset T_{0} \backslash W_{0}$, thus $T_{1}$ is contained in $T_{0} \backslash W_{0}$. Define inductively for $k \geq 1$,

$$
U_{k}=\phi\left(U_{k-1}\right), \quad V_{k}=\phi\left(V_{k-1}\right) \quad \text { and } \quad W_{k}=\phi\left(W_{k-1}\right)
$$

and define for $k \geq 0$, closed regions between $V_{k}$ and $V_{k+1}$

$$
\Theta_{k}=T_{k} \backslash\left(T_{k+1} \cup V_{k} \cup V_{k+1}\right),
$$

and solid tori

$$
t_{k}=T_{k} \backslash\left(V_{k} \backslash U_{k}\right) \text {. }
$$

Note that

$$
T_{k+1} \subset t_{k} \subset T_{k} .
$$

Fix a parameter $a \in(0,1)$. Define a metric $d_{a}$ on the Whitehead continuum $\mathbf{R}^{3} \backslash W h$ as follows. Initially, define a matrix $\left(\lambda_{i j}\right)=\operatorname{Id}$ on $\left(\mathbf{R}^{3} \backslash T_{0}\right) \cup W_{0}$ and define for $k \geq 1$,

$$
\left(\lambda_{i j}\right)=a^{2 k}\left(D \phi^{(-k)}\right)^{T} D \phi^{(-k)} \text { in } W_{k}
$$

where $\phi^{(-k)}$ is the $k$-th iterate of $\phi^{-1}$. Later, adjust $\left(\lambda_{i j}\right)$ in $\bigcup_{k=0}^{\infty}\left(W_{k} \backslash V_{k}\right)$ and extend $\left(\lambda_{i j}\right)$ to $\mathbf{R}^{3} \backslash W h$ so as to achieve the smoothness, the positive definiteness of $\left(\lambda_{i j}\right)$ and the self-similarity (3.3) below, and to preserve the previously defined values on $\left(\mathbf{R}^{3} \backslash T_{0}\right) \cup \bigcup_{k=0}^{\infty} V_{k}$. This can be accomplished by first extending $\left(\lambda_{i j}\right)$ continuously to $\Theta_{0} \backslash\left(W_{0} \cup W_{1}\right)$ with the aid of partition of unity, next smoothing $\left(\lambda_{i j}\right)$ in $\Theta_{0}$ by convolution with a kernel that varies with the Euclidean distance $\operatorname{dist}\left(x, \partial \Theta_{0}\right)$, and finally redefining $\left(\lambda_{i j}\right)$ in $\bigcup_{k=1}^{\infty} \Theta_{k}$ so that for each $k \geq 1$,

$$
\left(\lambda_{i j}\right)=a^{2 k}\left(D \phi^{(-k)}\right)^{T}\left(\lambda_{i j}\left(\phi^{(-k)}\right)\right) D \phi^{(-k)} \text { in } \Theta_{k} \text {. }
$$


The metric $d_{a}$ on $\mathbf{R}^{3} \backslash W h$ associated with the matrix $\left(\lambda_{i j}\right)$ is defined by $d_{a}(x, y)=\inf \{$ length $\gamma: \gamma$ continuously differentiable curve joining $x$ and $y\}$, where

$$
\text { length } \gamma=\int_{\gamma} \sqrt{\sum \lambda_{i j} d x_{i} d x_{j}}
$$

The metric thus defined is self-similar with respect to $W h$ in the following sense:

$$
d_{a}(x, y)=a^{k} \cdot d_{a}\left(\phi^{(-k)} x, \phi^{(-k)} y\right) \quad \text { for all } x \in T_{k^{\prime}} \text { and } y \in T_{k^{\prime \prime}}
$$

and for all $k^{\prime}, k^{\prime \prime} \geq k \geq \kappa \geq 0$ for some $\kappa$ depending only on $\phi$ and $c_{0}$.

The metric on the decomposition space $\mathbf{R}^{3} / W h$, again called $d_{a}$, is the pushforward metric induced by the homeomorphism $\pi: \mathbf{R}^{3} \backslash W h \rightarrow\left(\mathbf{R}^{3} / W h\right) \backslash\{q\}$ followed by the continuous extension to the point $q$.

Denote by $d_{e}$ be the Euclidean metric on $\mathbf{R}^{m}$ for any $m \geq 1$. Let $\delta_{a}$ be the metric on the product space $\left(\mathbf{R}^{3} / W h\right) \times \mathbf{R}^{m}$ defined by

$$
\delta_{a}\left(\left(x, x^{\prime}\right),\left(y, y^{\prime}\right)\right)=d_{a}(x, y)+d_{e}\left(x^{\prime}, y^{\prime}\right)
$$

for all $\left(x, x^{\prime}\right),\left(y, y^{\prime}\right) \in\left(\mathbf{R}^{3} / W h\right) \times \mathbf{R}^{m}$ and any $m \geq 1$.

Here, as well as in the future, we use $c, c_{1}, c_{2}, \ldots, C, C_{1}, C_{2}, \ldots, C^{\prime}, C^{\prime \prime}, \ldots$ to denote positive constants depending at most on $m, \phi, c_{0}$ and $a$, in particular, independent of $k$. We use $r \lesssim s, r \gtrsim s, r \simeq s$ to mean that the ratio $\frac{r}{s}$ is bounded above, or bounded below, or bounded above and below by this type of constants. Values of $c$ and $C$ need not be the same at each occurrence.

Note then

$$
\begin{aligned}
\operatorname{diam}_{d_{a}}\left(\widetilde{T}_{k}\right) & \simeq a^{k}, \\
\operatorname{dist}_{d_{a}}\left(\widetilde{t}_{k}, \partial \widetilde{T}_{k}\right) & =\operatorname{dist}_{d_{a}}\left(\widetilde{U}_{k}, \partial \widetilde{T}_{k}\right)=c_{0} a^{k}, \\
\operatorname{dist}_{d_{a}}\left(q, \partial \widetilde{T}_{k}\right) & \simeq \operatorname{dist}_{d_{a}}\left(\partial \widetilde{T}_{k}, \partial \widetilde{T}_{k+1}\right) \simeq \operatorname{dist}_{d_{a}}\left(\widetilde{V}_{k}, \widetilde{V}_{k+1}\right) \simeq a^{k} .
\end{aligned}
$$

We now give a concrete reformulation of Theorem 1.1.

3.8 Theorem The metric space $\left(\left(\mathbf{R}^{3} / W h\right) \times \mathbf{R}^{m}, \delta_{a}\right)$ is linearly locally contractible and Ahlfors $(3+m)$-regular. However there is no quasisymmetric homeomorphism between $\left(\left(\mathbf{R}^{3} / W h\right) \times \mathbf{R}^{m}, \delta_{a}\right)$ and $\mathbf{R}^{3+m}$, when $a>2^{-(3+m) / 2 m}$ and $m \geq 1$. 
The restriction on $a$ in the theorem is a consequence of the modulus estimates. It is unclear whether the theorem remains true for the whole range of $a \in(0,1)$.

The linear local contractibility and the Ahlfors regularity in Theorem 3.8 follow from (3.3)-(3.7) and a lifting-twisting-shrinking procedure used in proving the topological equivalence between $\left(\mathbf{R}^{3} / W h\right) \times \mathbf{R}^{m}$ and $\mathbf{R}^{3+m}$. By (3.3), (3.5), (3.6) and (3.7), there exists a large constant $C>1$ such that for each ball $B_{d_{a}}(x, r)$ in $\left(\mathbf{R}^{3} / W h, d_{a}\right)$, one of the following holds:

(i) $x=q$.

(ii) $x \in \widetilde{T}_{k} \backslash \widetilde{T}_{k+1}$ for some $k \geq-1$ and $q \in B_{d_{a}}(x, C r)$; in this case $B_{d_{a}}(x, r) \subset$ $B_{d_{a}}\left(q, C^{\prime} r\right) \subset B_{d_{a}}\left(x, C^{\prime \prime} r\right) \subset B_{d_{a}}\left(q, C^{\prime \prime \prime} r\right)$.

(iii) $x \in \widetilde{T}_{k} \backslash \widetilde{T}_{k+1}$ for some $k \geq-1$ and $q \notin B_{d_{a}}(x, C r)$; in this case $B_{d_{a}}(x, r) \subset$ $\widetilde{T}_{k-1} \backslash \widetilde{T}_{k+2}$ and there is an open set $U$ homeomorphic to a Euclidean ball so that

$$
B_{d_{a}}(x, r) \subset U \subset B_{d_{a}}(x, C r) .
$$

Here, with a slight abuse of notation, $\widetilde{T}_{-1}$ denotes $\mathbf{R}^{3} / W h$. By (3.4), there exists $C>1$ so that

$$
B_{\delta_{a}}\left((q,(0,0, \ldots, 0)), C^{-1} a^{k}\right) \subset \widetilde{T}_{k} \times\left[-a^{k}, a^{k}\right]^{m} \subset B_{\delta_{a}}\left((q,(0,0, \ldots, 0)), C a^{k}\right) .
$$

We first check that $\widetilde{T}_{1} \times[-1,1]^{m}$ is contractible inside $\widetilde{T}_{0} \times[-4,4]^{m}$. It is known that the quotient map $\pi \times$ id: $\mathbf{R}^{3+m} \rightarrow\left(\mathbf{R}^{3} / W h\right) \times \mathbf{R}^{m}$ can be uniformly approximated by homeomorphisms (see Daverman [9, pages 81-84]). Choose $g: \mathbf{R}^{3+m} \rightarrow\left(\mathbf{R}^{3} / W h\right) \times \mathbf{R}^{m}$, a homeomorphism satisfying

$$
\operatorname{dist}_{\delta_{a}}(g(x, z),(\pi \times \mathrm{id})(x, z))<c_{0} / 30 \text { on } \mathbf{R}^{3+m} .
$$

Then $g^{-1}\left(\widetilde{T}_{1} \times[-1,1]^{m}\right)$, a subset of $\left(T_{1} \cup V_{1}\right) \times[-2,2]^{m}$, is contractible inside $t_{0} \times[-3,3]^{m}$. Hence $\widetilde{T}_{1} \times[-1,1]^{m}$ is contractible in $g\left(t_{0} \times[-3,3]^{m}\right) \subset \widetilde{T}_{0} \times[-4,4]^{m}$. The linear local contractibility of $\left(\left(\mathbf{R}^{3} / W h\right) \times \mathbf{R}^{m}, \delta_{a}\right)$ can be seen by scaling and examining the projection of a given ball $B_{\delta_{a}}(x, r)$ into $\mathbf{R}^{3} / W h$ for the three alternative cases.

Define a measure $\mu$ on $\mathbf{R}^{3}=W h \cup\left(\mathbf{R}^{3} \backslash W h\right)$ as follows: $\mu(W h)=0, \mu \mid\left(\mathbf{R}^{3} \backslash T_{1}\right)$ is the Lebesgue measure, and

$$
\mu(E)=a^{3 k} \mu\left(\phi^{(-k)}(E)\right)
$$

if $E \subset T_{k} \backslash T_{k+1}$ and $k \geq 1$. The measure on the decomposition space $\left(\mathbf{R}^{3} / W h, d_{a}\right)$, again called $\mu$, is defined to be the pushforward of the measure $\mu$ on $\mathbf{R}^{3}$ induced by 
the quotient map $\pi: \mathbf{R}^{3} \rightarrow \mathbf{R}^{3} / W h$. Note from (3.3) that

$$
\mu\left(\widetilde{T}_{k} \backslash \widetilde{T}_{k+1}\right) \simeq a^{3 k} \text { for } k \geq 0,
$$

and that $\mu\left(B_{d_{a}}(q, r)\right) \simeq r^{3}$; and note from (3.3) and the smoothness of $\left(\lambda_{i j}\right)$ that $\mu\left(B_{d_{a}}(x, r)\right) \simeq r^{3}$ if $B_{d_{a}}(x, r) \subset \widetilde{T}_{k-1} \backslash \widetilde{T}_{k+2}$ and $k \geq 0$. The Ahlfors 3-regularity of $\left(\mathbf{R}^{3} / W h, d_{a}, \mu\right)$ follows by checking the three alternative cases. The product space $\left(\left(\mathbf{R}^{3} / W h\right) \times \mathbf{R}^{m}, \delta_{a}, \mu \times d x\right)$ is Ahlfors $(3+m)$-regular equipped with the product of $\mu$ with the Lebesgue measure $d x$ in $\mathbf{R}^{m}$.

\section{Modulus of surface families}

Let $f$ be a homeomorphism from $\left(\left(\mathbf{R}^{3} / W h\right) \times \mathbf{R}^{m}, \delta_{a}\right)$ onto $\mathbf{R}^{3+m}$. What is the obstruction for quasisymmetry? We give a heuristic explanation for $m=1$. Proposition 2.1 implies that $B \backslash \mathcal{L}$ is not simply connected for any small ball $B$ in $\left(\mathbf{R}^{3} / W h\right) \times \mathbf{R}^{1}$ that intersects $\mathcal{L}$. Hence $f(B) \backslash f(\mathcal{L})$ is not simply connected and $f(\mathcal{L})$ is wild in $\mathbf{R}^{4}$. A theorem of Martio, Rickman and Väisälä (Theorem 9.1 in the Appendix) then implies that the 2-dimensional Hausdorff measure

$$
\mathcal{H}^{2}(f(B) \cap f(\mathcal{L}))>0,
$$

for any ball $B$ in $\left(\mathbf{R}^{3} / W h\right) \times \mathbf{R}^{1}$ that intersects $\mathcal{L}$. One would then expect that $f$ maps all line segments of the form $\{z\} \times(t, t+s)$ in $\left(\mathbf{R}^{3} / W h\right) \times \mathbf{R}^{1}$ near $\{q\} \times \mathbf{R}^{1}$ to curves of much greater length. A comparison of moduli of suitable curve families in the domain and their images would prevent $f$ from being quasisymmetric. Nevertheless we are unable to provide quantitative estimates for the lengths of $f(\{z\} \times(t, t+s))$; so we shall work with the modulus of surface families instead.

The modulus of curve families has been an important tool in geometric function theory since the 1920's, first used by Grötzsch and Teichmüller and later extensively studied by Beurling and Ahlfors [1]. Extending the concept of modulus from curve families to $l$-dimensional surface families in $\mathbf{R}^{n}$ was due to Fuglede [15]. We need the notion of modulus for surface families in the setting of metric spaces. We shall not aim for full generality though.

Let $(X, d)$ be a linearly locally contractible topological $n$-manifold that is also Ahlfors $n$-regular. Let $1 \leq l \leq n-1, p$ a real number $\geq 1$ and $\mathcal{S}$ be a family of topological $l$-manifolds in $X$. A nonnegative Borel function $\rho: X \rightarrow[0, \infty]$ is called admissible for $\mathcal{S}$ if

$$
\int_{\Gamma} \rho(x) d \mathcal{H}_{d}^{l}(x) \geq 1
$$


for every $\Gamma \in \mathcal{S}$ that has positive Hausdorff $l$-measure $\mathcal{H}_{d}^{l}(\Gamma)$ and is locally finite with respect to $\mathcal{H}_{d}^{l}$. The $p$-modulus of $\mathcal{S}$ is defined by

$$
\operatorname{Mod}_{p} \mathcal{S}=\inf \int_{X} \rho^{p}(x) d \mathcal{H}_{d}^{n}(x),
$$

where the infimum is taken over all admissible $\rho: X \rightarrow[0, \infty]$. Here and later, we denote by $\mathcal{H}_{d}^{i}$ the $i$-th dimensional Hausdorff measure with respect to the metric $d$. We omit the subscript $d$ if the metric is the Euclidean one.

The most important modulus from the point of view of (quasi)conformal geometry is the $n / l$-modulus $\operatorname{Mod}_{n / l}$. It is conformally invariant and hence called the conformal modulus. The conformal modulus of curve families is quasi-invariant under quasisymmetric maps. This was proved by Gehring [16] for the Euclidean $n$-spaces and by Heinonen and Koskela [19] for very general metric spaces.

We consider a sequence of $(1+m)$-dimensional surface families $\mathcal{S}_{k}$ in $\left(\mathbf{R}^{3} / W h\right) \times \mathbf{R}^{m}$ defined as follows. First we select the curve family

$$
\mathcal{C}=\left\{\mathbf{S}^{1} \times\{x\}: x \in A\right\}
$$

in $T_{0} \subset \mathbf{R}^{3}$, where $A$ is the planar annulus defined in Section 3. Let

$$
\mathcal{C}_{k}=\left\{\pi \phi^{(k)}\left(\mathbf{S}^{1} \times\{x\}\right): x \in A\right\} \text { for } k \geq 0,
$$

be the associated curve families in the decomposition space $\mathbf{R}^{3} / W h$, then define the surface families

$$
\mathcal{S}_{k}=\left\{\gamma \times[0,1]^{m}: \gamma \in \mathcal{C}_{k}\right\} \text { for } k \geq 0,
$$

in $\left(\mathbf{R}^{3} / W h\right) \times \mathbf{R}^{m}$ by taking products.

We prove a one-sided inequality for the conformal moduli, which is adequate for our purpose. Constants depending on $f$ as well as $m, \phi, c_{0}$ and $a$, shall be denoted by $C(f)$.

4.1 Proposition Let $f$ be a quasisymmetric homeomorphism from $\left(\mathbf{R}^{3} / W h\right) \times \mathbf{R}^{m}$ onto $\mathbf{R}^{3+m}$. Then there exists a constant $C(f)>0$ such that

$$
\operatorname{Mod}_{(3+m) /(1+m)} \mathcal{S}_{k} \leq C(f) \operatorname{Mod}_{(3+m) /(1+m)} f\left(\mathcal{S}_{k}\right) \quad \text { for all } k \geq 0 .
$$

Proof Since the matrix $\left(\lambda_{i j}\right)$ is smooth in $\mathbf{R}^{3} \backslash W h$, the quasisymmetric map $f$ is in $W_{\text {loc }}^{1, r}\left(\left(\left(\mathbf{R}^{3} / W h\right) \times \mathbf{R}^{m}\right) \backslash \mathcal{L}\right)$ for some $r>3+m$; this can be seen by following the proof of the theorem of Gehring [17] on the higher integrability of the derivatives of quasiconformal maps in Euclidean spaces. Hence $f$ is differentiable $\mathcal{H}_{\delta_{a}}^{3+m}$-almost everywhere in $\left(\mathbf{R}^{3} / W h\right) \times \mathbf{R}^{m}$. Set, for $x \in A$,

$$
\Gamma_{x}=\pi \phi^{(k)}\left(\mathbf{S}^{1} \times\{x\}\right) \times[0,1]^{m} .
$$


In Euclidean spaces, quasiconformal mappings are absolutely continuous on almost all lines. Adapting the standard proof of this fact [36, Theorem 31.2], we may conclude that for $\mathcal{H}^{2}$-almost every $x \in A, f \mid \Gamma_{x}$ maps sets of $\mathcal{H}_{\delta_{a}}^{1+m}$-measure zero to sets of $\mathcal{H}^{1+m}$-measure zero. We may then conclude that for any nonnegative Borel function $\rho$ on $\mathbf{R}^{3+m}$ and for $\mathcal{H}^{2}$-almost every $x \in A$,

$$
\int_{\Gamma_{x}} \rho(f(x))|D f(x)|^{1+m} d \mathcal{H}_{\delta_{a}}^{1+m}(x) \geq \int_{f\left(\Gamma_{x}\right)} \rho(y) d \mathcal{H}^{1+m}(y),
$$

by adapting the standard argument for quasiconformal maps [36, Theorem 5.3]. Quasisymmetry implies that

$$
\begin{aligned}
\int_{\left(\mathbf{R}^{3} / W h\right) \times \mathbf{R}^{m}} & \left(\rho(f(x))|D f(x)|^{1+m}\right)^{(3+m) /(1+m)} d \mathcal{H}_{\delta_{a}}^{3+m}(x) \\
& \leq C(f) \int_{\left(\mathbf{R}^{3} / W h\right) \times \mathbf{R}^{m}} \rho(f(x))^{(3+m) /(1+m)}|\operatorname{det} D f(x)| d \mathcal{H}_{\delta_{a}}^{3+m}(x) \\
& \leq C(f) \int_{\mathbf{R}^{3+m}} \rho(y)^{(3+m) /(1+m)} d \mathcal{H}^{3+m}(y) .
\end{aligned}
$$

From the fact that the union of these surfaces has $\mathcal{H}_{\delta_{a}}^{3+m}$-measure zero, it follows that the exceptional surfaces for which (4.3) fails have vanishing conformal modulus. Then (4.2) can be obtained by following the standard argument for modulus of curve families (see Theorem 32.3 in [36] or Theorem II.2.4 in [26]).

We need the following estimates.

4.4 Proposition Let $f$ be a homeomorphism from $\left(\mathbf{R}^{3} / W h\right) \times \mathbf{R}^{m}$ onto $\mathbf{R}^{3+m}$. Then for each $k \geq 0$ and each $\Gamma \in \mathcal{S}_{k}$, the $(1+m)$-dimensional Hausdorff measures in $\left(\left(\mathbf{R}^{3} / W h\right) \times \mathbf{R}^{m}, \delta_{a}\right)$ and $\mathbf{R}^{3+m}$ satisfy

(1) $\mathcal{H}_{\delta_{a}}^{1+m}(\Gamma) \simeq a^{k}$,

(2) $\mathcal{H}^{1+m}(f(\Gamma)) \geq C(f) 2^{k}$

for some positive constant $C(f)$.

4.5 Proposition Let $f$ be a homeomorphism from $\left(\mathbf{R}^{3} / W h\right) \times \mathbf{R}^{m}$ onto $\mathbf{R}^{3+m}$. Then for each $k \geq 0$, the conformal moduli of surface families satisfy

(1) $\operatorname{Mod}_{(3+m) /(1+m)} \mathcal{S}_{k} \simeq\left(a^{k}\right)^{2 m /(1+m)}$,

(2) $\operatorname{Mod}_{(3+m) /(1+m)} f\left(\mathcal{S}_{k}\right) \leq C(f)\left(2^{-k}\right)^{(3+m) /(1+m)}$

for some positive constant $C(f)$. 


\section{Proof of Theorem 1.1}

Theorem 1.1 follows from Theorem 3.8. The last sentence of Theorem 3.8, the only statement yet to be proved, follows from Proposition 4.1 and Proposition 4.5. The proof of Proposition 4.5 is given first assuming the validity of Proposition 4.4. The proof of Proposition 4.4 is given next assuming the validity of an estimate (5.1) on the intersection numbers. The proof of (5.1) shall occupy the remaining sections.

Proof of Proposition 4.5 Assume the validity of Proposition 4.4. Fix $k \geq 0$ and let $\rho$ be a nonnegative Borel function on $\left(\mathbf{R}^{3} / W h\right) \times \mathbf{R}^{m}$ admissible for the surface family $\mathcal{S}_{k}$, thus

$$
\int_{\Gamma} \rho d \mathcal{H}_{\delta_{a}}^{1+m} \geq 1
$$

for every $\Gamma \in \mathcal{S}_{k}$. Therefore

$$
\begin{aligned}
\int_{\tilde{U}_{k} \times[0,1]^{m}} \rho d \mathcal{H}_{\delta_{a}}^{3+m} & \simeq \int_{\mathbf{S}^{1} \times A}\left(\int_{[0,1]^{m}} \rho\left(\pi \phi^{(k)}(s, x), z\right) d \mathcal{H}^{m}(z)\right) a^{3 k} d \mathcal{H}^{3}(s, x) \\
& \simeq \int_{A}\left(\int_{\pi \phi^{(k)}\left(\mathbf{S}^{1} \times\{x\}\right)}\left(\int_{[0,1]^{m}} \rho(\xi, z) d \mathcal{H}^{m}(z)\right) d \mathcal{H}_{d_{a}}^{1}(\xi)\right) a^{2 k} d \mathcal{H}^{2}(x) \\
& \simeq \int_{A}\left(\int_{\pi \phi^{(k)}\left(\mathbf{S}^{1} \times\{x\}\right) \times[0,1]^{m}} \rho(\xi, z) d \mathcal{H}_{\delta_{a}}^{1+m}(\xi, z)\right) a^{2 k} d \mathcal{H}^{2}(x) \gtrsim a^{2 k} .
\end{aligned}
$$

On the other hand,

$$
\begin{aligned}
& \int_{\widetilde{U}_{k} \times[0,1]^{m}} \rho d \mathcal{H}_{\delta_{a}}^{3+m} \\
& \leq\left(\int_{\widetilde{U}_{k} \times[0,1]^{m}} \rho^{(3+m) /(1+m)} d \mathcal{H}_{\delta_{a}}^{3+m}\right)^{(1+m) /(3+m)}\left(\mathcal{H}_{\delta_{a}}^{3+m}\left(\widetilde{U}_{k} \times[0,1]^{m}\right)\right)^{2 /(3+m)} \\
& \leq C\left(\int_{\widetilde{U}_{k} \times[0,1]^{m}} \rho^{(3+m) /(1+m)} d \mathcal{H}_{\delta_{a}}^{3+m}\right)^{(1+m) /(3+m)} a^{6 k /(3+m)} .
\end{aligned}
$$

This implies

$$
\int_{\widetilde{U}_{k} \times[0,1]^{m}} \rho^{(3+m) /(1+m)} d \mathcal{H}_{\delta_{a}}^{3+m} \gtrsim\left(a^{k}\right)^{2 m /(1+m)} .
$$

Now set $\rho_{0}=a^{-k}$ in $\widetilde{U}_{k} \times[0,1]^{m}$ and $=0$ outside $\tilde{U}_{k} \times[0,1]^{m}$. Note from Proposition 4.4(1) that $C \rho_{0}$ is admissible for the surface family $\mathcal{S}_{k}$ for some constant $C>0$ 
depending at most on $m, \phi, c_{0}$ and $a$, and in particular independent of $k$. It is easy to check that

$$
\int_{\left(\mathbf{R}^{3} / W h\right) \times \mathbf{R}^{m}} \rho_{0}^{(3+m) /(1+m)} d \mathcal{H}_{\delta_{a}}^{3+m} \simeq\left(a^{-k}\right)^{(3+m) /(1+m)}\left(a^{k}\right)^{3} \simeq\left(a^{k}\right)^{2 m /(1+m)} .
$$

Therefore

$$
\operatorname{Mod}_{(3+m) /(1+m)} \mathcal{S}_{k} \simeq\left(a^{k}\right)^{2 m /(1+m)} .
$$

To show part (2), set $\rho_{1}=2^{-k}$ in $f\left(\tilde{U}_{k} \times[0,1]^{m}\right)$ and $\rho_{1}=0$ in $\mathbf{R}^{3+m} \backslash f\left(\tilde{U}_{k} \times[0,1]^{m}\right)$. Note from Proposition $4.4(2)$ that $C(f) \rho_{1}$ is admissible for $f\left(\mathcal{S}_{k}\right)$ for some $C(f)>0$. Then

$$
\int_{\mathbf{R}^{3+m}} \rho_{1}^{(3+m) /(1+m)} d \mathcal{H}^{3+m} \leq C(f) \mathcal{H}^{3+m}\left(f\left(\widetilde{T}_{0} \times[0,1]^{m}\right)\right)\left(2^{-k}\right)^{(3+m) /(1+m)} .
$$

Hence $\operatorname{Mod}_{(3+m) /(1+m)} f\left(\mathcal{S}_{k}\right) \leq C(f)\left(2^{-k}\right)^{(3+m) /(1+m)}$.

This completes the proof.

Proof of Proposition 4.4 Part (1) follows immediately from the definition of the metric $\delta_{a}$.

To prove part (2), we first construct a bi-Lipschitz homeomorphism $\chi$ of $\mathbf{R}^{3+m}$ with some specific properties, we then slice surfaces in the collection $\bigcup_{k=k_{0}}^{\infty}\left(\chi \circ f\left(\mathcal{S}_{k}\right)\right)$ by a certain stack of standard 2-simplices $\left\{D_{j}\right\}$ in $\mathbf{R}^{3+m}$ with the parameter $j$ ranging over an $(1+m)$-dimensional cube $J$ in $\mathbf{R}^{3+m}$ orthogonal to the simplices $\left\{D_{j}\right\}$. Finally we estimate the number $N(k, \Gamma, j)$ of points in the intersection $D_{j} \cap(\chi \circ f(\Gamma))$ from below by

$$
N(k, \Gamma, j) \geq C(f) 2^{k} \quad \text { for all } k>k_{0}, \Gamma \in \mathcal{S}_{k} \text { and } j \in J,
$$

for some positive integer $k_{0}$ and some positive constant $C(f)$ depending on $f$.

Assume that ((5.1)) is valid for the moment. Given $k>k_{0}$ and a surface $\Gamma$ in $\mathcal{S}_{k}$, let $\zeta$ be the projection map from $\left(\bigcup_{j \in J} D_{j}\right) \cap(\chi \circ f(\Gamma))$ to $J$ defined by

$$
\zeta(x)=j \quad \text { if } x \in D_{j} \cap(\chi \circ f(\Gamma)) .
$$

So $\zeta$ is 1 -Lipschitz. It follows from (5.1) and a coarse estimate (see Federer [13, Theorem 2.10.25]) that

$$
\mathcal{H}^{1+m}(\chi \circ f(\Gamma)) \geq \int_{J} N(k, \Gamma, j) d \mathcal{H}^{1+m}(j) \geq C(f) \mathcal{H}^{1+m}(J) 2^{k} .
$$

Since $\chi$ is bi-Lipschitz, part (2) has been proved assuming (5.1). 
To complete the proof of Theorem 1.1, it remains to establish (5.1). In Section 6 we define $\chi$ and locate the stack of two-simplices used for slicing, and in Section 8 we prove the lower estimate (5.1).

\section{A stack of two-simplices}

Denote by $B_{d}(x, r)$ the closed ball having center $x$ and radius $r$ in the metric space $(X, d)$. We omit the subscript when the metric is Euclidean.

Let 0 be the origin of $\mathbf{R}^{m}$ and $Q$ be the point $(q, 0)$ in $\left(\mathbf{R}^{3} / W h\right) \times \mathbf{R}^{m}$. Recall from (3.5), (3.6) and (3.7) that $q \in \widetilde{T}_{k} \subset \widetilde{T}_{k} \cup \widetilde{V}_{k} \subset B_{d_{a}}\left(q, C a^{k}\right) \subset \mathbf{R}^{3} / W h$ and that

$$
Q \in\left(\widetilde{T}_{k} \cup \tilde{V}_{k}\right) \times\left[-a^{k}, a^{k}\right]^{m} \subset B_{\delta_{a}}\left(Q, C_{1} a^{k}\right),
$$

for some constants $C, C_{1}>1$. Set

$$
v_{k}=\operatorname{diam} f\left(B_{\delta_{a}}\left(Q, C_{1} a^{k}\right)\right) ;
$$

choose and fix an integer $k_{0} \geq 2$ so that

$$
B\left(f(Q), 3 v_{k_{0}}\right) \subset f\left(\widetilde{T}_{1} \times \mathbf{R}^{m}\right) .
$$

The 2-simplices shall be chosen to lie in $B\left(f(Q), 3 v_{k_{0}}\right)$.

Set

$$
\alpha=\pi \phi^{\left(k_{0}\right)}\left(\partial B_{0}^{2}\right) \times\{0\},
$$

a meridian of $\widetilde{T}_{k_{0}} \times\{0\}$, where $B_{0}^{2}$ is the planar disk in $\mathbf{R}^{3}$ defined in the beginning of Section 2. Let

$$
\mathfrak{A}=\left\{x \in \mathbf{R}^{3} / W h: \operatorname{dist}_{\delta_{a}}((x, 0), \alpha)<\frac{c_{0} a^{k_{0}}}{30}\right\} \times\left(-\frac{c_{0} a^{k_{0}}}{30}, \frac{c_{0} a^{k_{0}}}{30}\right)^{m}
$$

be a tubular neighborhood of $\alpha$ not intersecting $\widetilde{t}_{k_{0}} \times \mathbf{R}^{m}$, where $c_{0}$ is the constant chosen in Section 3.

Let $c_{1}=\operatorname{dist}(f(\alpha), f(\partial \mathfrak{A})) / 10$. It follows from a PL-approximation theorem of Moise (Theorem 9.2 in the Appendix) that there exists an embedding $\theta: \alpha \hookrightarrow \mathbf{R}^{3+m}$ such that $l \equiv \theta(\alpha)$ is a piecewise linear topological 1-sphere in $f(\mathfrak{A})$, and

$$
d(f(x), \theta(x))<c_{1} \text { for all } x \in \alpha .
$$

The embedding $\theta$ used for defining $l$ shall play no role in the future. We observe that $l$ and $f(\alpha)$ are homotopic in $f(\mathfrak{A})$ and that

$$
\begin{aligned}
l \subset f(\mathfrak{A}) & \subset f\left(\left(\tilde{T}_{k_{0}} \cup \tilde{V}_{k_{0}}\right) \times\left[-a^{k_{0}}, a^{k_{0}}\right]^{m}\right) \\
& \subset f\left(B_{\delta_{a}}\left(Q, C_{1} a^{k_{0}}\right)\right) \subset B\left(f(Q), v_{k_{0}}\right) .
\end{aligned}
$$


Here, as well as in the future, two curves $\beta$ and $\beta^{\prime}$ are said to be homotopic in $X$ (or $\beta$ is homotopic to $\beta^{\prime}$ in $X$ ) if the continuous maps defining the curves are homotopic in the target space $X$.

Since $3+m \geq 4$, the PL 1 -sphere $l$ is unknotted in $\mathbf{R}^{3+m}$ and there is a global bi-Lipschitz homeomorphism $\chi$ of $\mathbf{R}^{3+m}$ that maps $l$ to the boundary of a standard 2-simplex $D^{\prime}$ contained in $B(Y, 2 \operatorname{diam} l), \chi(l)=\partial D^{\prime}$, while fixes all points outside $B(Y, 2 \operatorname{diam} l)$ for some point $Y \in l$. (See Theorem 9.4 in the Appendix.) Note from (6.3) that

$$
\chi=\text { id in } \mathbf{R}^{3+m} \backslash B\left(f(Q), 3 v_{k_{0}}\right) .
$$

Choose and fix a $(1+m)$-dimensional cube $J$ in $\mathbf{R}^{3+m}$ which is orthogonal to the 2simplex $D^{\prime}$ and is small enough such that all translates $j+\partial D^{\prime}(j \in J)$ are contained in $\chi \circ f(\mathfrak{A})$ and are homotopic to $\chi \circ f(\alpha)$ in $\chi \circ f(\mathfrak{A})$. The translated standard 2-simplices $D_{j} \equiv j+D^{\prime}, j \in J$ enclosed by $j+\partial D^{\prime}$ shall be used for slicing surfaces in the collection $\bigcup_{k=k_{0}}^{\infty}\left(\chi \circ f\left(\mathcal{S}_{k}\right)\right)$.

Since $\partial D_{j}=j+\partial D^{\prime} \subset \chi \circ f(\mathfrak{A}) \subset \chi\left(B\left(f(Q), v_{k_{0}}\right)\right) \subset B\left(f(Q), 3 v_{k_{0}}\right)$, it follows that

$$
D_{j} \subset B\left(f(Q), 3 v_{k_{0}}\right) .
$$

From (6.1) and (6.4), it follows that

$$
f^{-1} \chi^{-1}\left(D_{j}\right) \subset \widetilde{T}_{1} \times \mathbf{R}^{m}, \text { for all } j \in J .
$$

Since $f^{-1} \chi^{-1}\left(\partial D_{j}\right)$ and $\alpha$ are homotopic in $\mathfrak{A}, \alpha$ is a meridian of $\widetilde{T}_{k_{0}} \times\{0\}$ and $\mathfrak{A} \cap \mathcal{L}=\varnothing$, the curve $f^{-1} \chi^{-1}\left(\partial D_{j}\right)$ is not contractible in $\left(\widetilde{T}_{0} \times \mathbf{R}^{m}\right) \backslash \mathcal{L}$ by Proposition 2.1. Therefore

$$
f^{-1} \chi^{-1}\left(D_{j}\right) \cap \mathcal{L} \neq \varnothing, \text { for all } j \in J \text {. }
$$

6.5 Remark For simplicity and with a slight abuse of notation, we continue to use the notation $f$ for the composition map $\chi \circ f$. Under this convention, for every $j \in J$,

$$
\partial D_{j} \subset f(\mathfrak{A})
$$

$f^{-1}\left(\partial D_{j}\right)$ and $\alpha$ are homotopic in $\mathfrak{A}$; and

$$
f^{-1}\left(D_{j}\right) \cap \mathcal{L} \neq \varnothing, D_{j} \cap f(\mathcal{L}) \neq \varnothing \quad \text { and } \quad f^{-1}\left(D_{j}\right) \subset \widetilde{T}_{1} \times\left[-C_{2}, C_{2}\right]^{m}
$$

for some $C_{2}>1$. 


\section{Interior-essential components}

The tori $T_{k}(k \geq 0)$ used in the Whitehead construction can be arranged topologically so that the meridian $\partial B_{0}^{2}$ on $\partial T_{0}$ bounds a $2-$ cell in $T_{0}$ which intersects every $T_{k}$ $(k \geq 1)$ in $2^{k}$ mutually disjoint 2 -cells enclosed by meridians of $T_{k}$. The lower estimate (5.1) is suggested by this idealized setting followed by a homological argument of Freedman and Skora [14]. In this section, we prove a version of their Lemma 2.5 for mappings instead of embeddings.

We adopt some statements from [9, pages 73-74] in locating the essential part of a continuous image of a 2-cell inside an $n$-manifold. Let $M$ be an $n$-manifold with boundary and $\omega$ a 2 -manifold with boundary. A map $\Phi:(\omega, \partial) \rightarrow(M, \partial)$, meaning $\Phi(\omega) \subset M$ and $\Phi(\partial \omega) \subset \partial M$, is said to be interior-inessential if there exists a map $\Phi^{\prime}: \omega \rightarrow \partial M$ satisfying $\Phi^{\prime}|\partial \omega=\Phi| \partial \omega$; it is said to be interior-essential if no such $\Phi^{\prime}$ exists. In case $\omega$ is a closed 2-cell, $\Phi$ is interior-essential if and only if $\Phi(\partial \omega)$ is homotopically nontrivial in $\partial M$, but trivial in $M$.

Suppose that $\omega$ is a compact, connected 2-manifold in a 2-cell $D$. Denote by $D_{\omega}$ the unique $2-$ cell in $D$ containing $\omega$ with $\partial D_{\omega} \subset \partial \omega$. A map $\Phi:(\omega, \partial) \rightarrow(M, \partial)$ is said to be virtually interior-essential if $\Phi$ extends to an interior-essential $\Phi^{\prime \prime}: D_{\omega} \rightarrow M$ satisfying $\Phi^{\prime \prime}\left(D_{\omega} \backslash \omega\right) \subset \partial M$.

In case $M$ is a solid torus $T=\mathbf{S}^{1} \times B^{2}$, an interior-essential map $\Phi:(\omega, \partial) \rightarrow(T, \partial)$ necessarily maps one of the boundary components of $\omega$ to a homotopically nontrivial loop on $\partial T$. A map $\Phi:(\omega, \partial) \rightarrow(T, \partial)$ is virtually interior-essential if and only if $\Phi$ maps the outermost component of $\partial \omega$ to a nonzero multiple of a meridian on $\partial T$ while mapping all other components of $\partial \omega$ to trivial loops there. In other words $\Phi$, in fact the extension $\Phi^{\prime \prime} \mid D_{\omega}$, represents a nonzero multiple of a generator of the relative homology group $H_{2}(T, \partial ; \mathbb{Z}) \equiv \mathbb{Z}$. Since a meridian and a core of a torus are linked, $\Phi(\omega)$ must intersect every core of the torus $T$.

Here and later, a closed curve on $\partial T$ is said to be a multiple of a meridian if it is homotopic, in $\partial T$, to $n$ times a meridian of $T$ for some integer $n$. A boundary component of a compact 2-manifold $\omega$ in a 2-cell $D$ is said to be outermost if it is not contained in the 2-cell in $D$ enclosed by any other component of $\partial \omega$.

Recall that the relative homology group

$$
H_{2}\left(\mathbf{S}^{1} \times B^{2}, \partial ; \mathbb{Z}\right) \equiv \mathbb{Z}
$$

is generated by the equivalence class $[g]$ with $g:(\Delta, \partial \Delta) \rightarrow\left(\mathbf{S}^{1} \times B^{2}, \partial\left(\mathbf{S}^{1} \times B^{2}\right)\right)$ being continuous and of degree \pm 1 on $\partial \Delta$, where $\Delta$ is a standard 2 -simplex.

The following observation on meridians and longitudes is needed. 
7.1 Lemma Let $T_{k}(k \geq 0)$ be the solid tori in the Whitehead construction. Then the meridians of $T_{k}$ are not contractible in $\mathbf{S}^{3} \backslash \operatorname{Int}\left(T_{k}\right)$ hence not in $T_{0} \backslash \operatorname{Int}\left(T_{k}\right)$, and the longitudes of $T_{k}$ are not contractible in $T_{0} \backslash \operatorname{Int}\left(T_{k}\right)$.

Proof The noncontractibility of the meridians follows from the fact that a meridian (resp. a longitude) of the torus $T$ is a longitude (resp. a meridian) of the torus $\mathbf{S}^{3} \backslash \operatorname{Int}(T)$.

Since a core of $T_{k}$ and a core of $\mathbf{S}^{3} \backslash \operatorname{Int}\left(T_{k-1}\right)$ form a Whitehead link for $k \geq 1$, there is a homeomorphism $h$ of $\mathbf{S}^{3}$ interchanging these two cores [21, page 83]. In fact, $h$ can be chosen to map $T_{k}$ and $\mathbf{S}^{3} \backslash \operatorname{Int}\left(T_{k-1}\right)$ onto $\mathbf{S}^{3} \backslash \operatorname{Int}\left(T_{k-1}\right)$ and $T_{k}$ respectively, thus to map $T_{k-1}$ onto $\mathbf{S}^{\mathbf{3}} \backslash \operatorname{Int}\left(T_{k}\right)$. This implies that $\mathbf{S}^{\mathbf{3}} \backslash \operatorname{Int}\left(T_{k-1}\right)$ is embedded in $\mathbf{S}^{3} \backslash \operatorname{Int}\left(T_{k}\right)$ in same way that $T_{k}$ is embedded in $T_{k-1}$ for all $k \geq 1$. From this and the fact mentioned in the last paragraph, the statement on the noncontractibility of the longitudes follows.

We now prove an analogue of Lemma 2.5 in Freedman and Skora [14] for mappings; it was proved there for embeddings and for the Bing double.

7.2 Lemma Let $T_{0}$ and $T_{1}$ be the first two tori in the Whitehead construction, $\omega$ be a compact, connected 2-manifold in a 2-cell $D$, and $\Phi:(\omega, \partial) \rightarrow\left(T_{0}, \partial\right)$ be a virtually interior-essential map. Suppose that $\Phi(\omega)$ meets $\partial T_{0} \cup \partial T_{1}$ transversally. Then $\Phi^{-1}\left(T_{1}\right)$ contains at least two components $\omega^{1}, \omega^{2}$ on which the maps $\Phi \mid \omega^{i}: \omega^{i} \rightarrow$ $T_{1}(i=1,2)$ are virtually interior-essential.

Proof Assume that $\Phi$ has been extended to the 2-cell $D_{\omega}$, which maps $\partial D_{\omega}$ to a nonzero multiple of a meridian of $T_{0}$ and maps $D_{\omega} \backslash \omega$ into $\partial T_{0}$.

We first check that $\left[\Phi \mid \Phi^{-1}\left(T_{1}\right), \partial\right]=0$ in $H_{2}\left(T_{1}, \partial ; \mathbb{Z}\right)$, an analogue of an assertion of Freedman and Skora about the Bing double [14, page 82]. Choose a smooth embedding $g:(\Delta, \partial \Delta, \Delta \backslash \partial \Delta) \hookrightarrow\left(T_{0}, \partial T_{0}, T_{0} \backslash \partial T_{0}\right)$ in such a way that $g(\Delta)$ meets $\partial T_{1}$ transversally, $g^{-1}\left(T_{1}\right)$ consists of exactly two 2 -cells $\Delta_{i}(i=1,2),[g, \partial]$ generates $H_{2}\left(T_{0}, \partial ; \mathbb{Z}\right)$, and $g \mid \Delta_{1}$ and $g \mid \Delta_{2}$ represent generators of $H_{2}\left(T_{1}, \partial ; \mathbb{Z}\right)$ of opposite signs. Hence

$$
\left[g \mid \Delta_{1}, \partial\right]+\left[g \mid \Delta_{2}, \partial\right]=0 \text { in } H_{2}\left(T_{1}, \partial ; \mathbb{Z}\right) .
$$

Identify the 2-cells $D_{\omega}, \Delta_{1}$ and $\Delta_{2}$ with the standard 2-simplex $\Delta$ with proper orientations. Since

$$
[\Phi, \partial]= \pm n[g, \partial] \text { in } H_{2}\left(T_{0}, \partial ; \mathbb{Z}\right),
$$


for some 3-chain $\sigma$ in $T_{0}$ and some 2-chain $\tau$ in $\partial T_{0}$. After subdivision, all chains in (7.3) are sums of singular simplices in $T_{1}$ or $T_{0} \backslash \operatorname{Int}\left(T_{1}\right)$. Since the group $H_{2}\left(T_{0}, T_{0} \backslash \operatorname{Int}\left(T_{1}\right) ; \mathbb{Z}\right)$ is isomorphic to $H_{2}\left(T_{1}, \partial ; \mathbb{Z}\right)$ by excision, [ $\left.\Phi\right]=$ $\left[\Phi \mid \Phi^{-1}\left(T_{1}\right), \partial\right],[g]=\left[g \mid \Delta_{1}, \partial\right]+\left[g \mid \Delta_{2}, \partial\right]=0,[\partial \sigma]=\left[\partial \sigma^{\prime}\right]=0$ for a $3-$ chain $\sigma^{\prime}$ in $T_{1}$, and $[\tau]=0$ in $H_{2}\left(T_{1}, \partial ; \mathbb{Z}\right)$. Therefore $\left[\Phi \mid \Phi^{-1}\left(T_{1}\right), \partial\right]=0$ in $H_{2}\left(T_{1}, \partial ; \mathbb{Z}\right)$.

By transversality and the Schönflies Theorem, $\Phi^{-1}\left(T_{1}\right)$ is a compact $2-$ manifold in $D_{\omega}$ and $\Phi^{-1}\left(\partial T_{1}\right)$ is a 1 -manifold with no boundary. Moreover $\Phi^{-1}\left(T_{1}\right)$ is nonempty and contains some component on which $\Phi$ is interior-essential. Otherwise, $\Phi \mid \Phi^{-1}\left(T_{1}\right)$ could be redefined in the interior-inessential components to have images in $\partial T_{1}$; consequently, $\Phi\left(D_{\omega}\right) \subset T_{0} \backslash \operatorname{Int}\left(T_{1}\right)$. This would imply that $\Phi\left(\partial D_{\omega}\right)$, a nonzero multiple of a meridian of $T_{0}$, is contractible in $T_{0} \backslash \operatorname{Int}\left(T_{1}\right)$.

Among the components of $\Phi^{-1}\left(T_{1}\right)$ on which $\Phi$ is interior-essential, (call the entire collection of such components $\Omega$ ), choose an innermost component $\omega^{1}$; ie, $D_{\omega^{1}}$ contains no element in $\Omega \backslash\left\{\omega^{1}\right\}$. We claim that $\Phi \mid \omega^{1}$ is virtually interior-essential. To verify the claim, note that the boundary of $\omega^{1}$ consists of a special outermost component $\partial D_{\omega^{1}}$ and possibly some other components $\beta$ 's whose interior contains no component of $\Phi^{-1}\left(T_{1}\right)$ on which $\Phi$ is interior-essential. Modify $\Phi$ on the interiorinessential components inside $\beta$, so that it maps the $2-$ cell $D_{\beta}$ in $D_{\omega}$ enclosed by $\beta$ into $T_{0} \backslash \operatorname{Int}\left(T_{1}\right)$. This implies that $\Phi(\beta)$ is contractible in $T_{0} \backslash \operatorname{Int}\left(T_{1}\right)$. Neither the meridians of $T_{1}$ nor the longitudes of $T_{1}$ are contractible in $T_{0} \backslash \operatorname{Int}\left(T_{1}\right)$ by Lemma 7.1; $\Phi(\beta)$ must be homotopically trivial in $\partial T_{1}$. Assume as we may that $\Phi\left(D_{\beta}\right) \subset \partial T_{1}$. Since $\Phi \mid \omega^{1}$ is interior-essential, $\Phi\left(\partial D_{\omega^{1}}\right)$ must be nontrivial in $\partial T_{1}$. Since $\Phi\left(\partial D_{\omega^{1}}\right)$ $\left(\subset \Phi\left(D_{\omega^{1}}\right) \subset T_{0}\right)$ is contractible in $T_{0}$, it has to be a nonzero multiple, say $n_{1}$, of a meridian of $T_{1}$. This proves the claim.

Assume as we may that $\omega^{1}=D_{\omega^{1}}$. Therefore $\Phi \mid \omega^{1}$ represents a nonzero multiple of a generator of the relative homology group $H_{2}\left(T_{1}, \partial ; \mathbb{Z}\right)$. Since $\left[\Phi \mid \Phi^{-1}\left(T_{1}\right), \partial\right]=0$ in $H_{2}\left(T_{1}, \partial ; \mathbb{Z}\right), \Omega \backslash\left\{\omega^{1}\right\}$ is nonempty. Suppose that $\omega^{2}\left(\neq \omega^{1}\right)$ is another innermost component of $\Omega$, then $\Phi \mid \omega^{2}: \omega^{2} \rightarrow T_{1}$ must also be virtually interior-essential.

Suppose $\omega^{1}$ is the only innermost component of $\Omega$. We shall prove $\left[\Phi \mid \Phi^{-1}\left(T_{1}\right), \partial\right] \neq 0$. This leads to a contradiction, thus the validity of the lemma.

The assumption implies that the minimal 2-cells $D_{\omega^{\prime}}$ in $D_{\omega}$ containing $\omega^{\prime} \quad\left(\omega^{\prime} \in \Omega\right)$ are nested and contain $\omega^{1}$ in their intersection. In other words, the elements of $\Omega$, $\omega^{1}, \omega^{2}, \ldots, \omega^{N}$, can be ordered so that $\omega^{1}=D_{\omega^{1}} \subset D_{\omega^{2}} \subset \cdots \subset D_{\omega^{N}} \subset D_{\omega}$.

We shall alter $\Phi$ in such a way, while not changing the homology class $\left[\Phi \mid \Phi^{-1}\left(T_{1}\right), \partial\right]$ in $H_{2}\left(T_{1}, \partial ; \mathbb{Z}\right)$, that $\Phi^{-1}\left(T_{1}\right)$ has only one interior-essential component, namely $D_{\omega^{N}}$. Since $\Phi\left(\partial D_{\omega}\right)$ is noncontractible in $T_{0} \backslash \operatorname{Int}\left(T_{1}\right), \Phi\left(\partial D_{\omega^{N}}\right)$ must be homotopically nontrivial in $\partial T_{1}$. It follows then $\left[\Phi \mid \Phi^{-1}\left(T_{1}\right), \partial\right]=\left[\Phi \mid D_{\omega^{N}}, \partial\right] \neq 0$. 
The boundary of $\omega^{2}$ consists an outermost component $\partial D_{\omega^{2}}$ and a special component $b$ that encloses a $2-$ cell $D_{b}$ in $D_{\omega}$ which contains $\omega^{1}$ in its interior, and possibly some other components $\beta$ 's whose interiors contain no elements of $\Omega$. Since $D_{\beta}$ does not contain any element of $\Omega, \Phi(\beta)$ is trivial in $\partial T_{1}$. After altering $\Phi$ in the interior of each $D_{\beta}$, we may assume that $\bigcup_{\beta} \Phi\left(D_{\beta}\right) \subset \partial T_{1}$ and that $\Phi$ maps the annular region in $D_{\omega}$ bounded by $b$ and $\partial D_{\omega^{2}}$ into $T_{1}$. The 2-cell $D_{b}$ consists of $D_{\omega^{1}}$ and an annular region $\mathcal{A}$ in $D_{\omega}$ bounded by $\partial D_{\omega^{1}}$ and $b$. The annulus $\mathcal{A}$ contains no elements of $\Omega$ due to the ordering. Modifications can be made on the interior-inessential components in $\mathcal{A}$, so that $\Phi \mid \mathcal{A}$ maps $\mathcal{A}$ into $T_{0} \backslash \operatorname{Int}\left(T_{1}\right)$. The annulus $\mathcal{A}$ provides a homotopy between $\Phi \mid b$ and $\Phi \mid \partial D_{\omega^{1}}$ in $T_{0} \backslash \operatorname{Int}\left(T_{1}\right)$. By homotopy invariance, both $\Phi(b)$ and $\Phi\left(\partial D_{\omega^{1}}\right)$ are $n_{1}$ times a meridian of $T_{1}$. So $\Phi$ can again be altered in $\mathcal{A} \backslash \Phi^{-1}\left(T_{1}\right)$ such that $\Phi(\mathcal{A}) \subset \partial T_{1}$. After these changes, $D_{\omega^{2}}$ becomes a component of $\Phi^{-1}\left(T_{1}\right)$ while the homological relation $\left[\Phi \mid \omega^{1}, \partial\right]+\left[\Phi \mid \omega^{2}, \partial\right]=\left[\Phi \mid D_{\omega^{2}}, \partial\right]$ remains.

Continuing inductively if $N>2, \Phi$ can be altered so that $D_{\omega^{N}}$ becomes the only interior-essential component of $\Phi^{-1}\left(T_{1}\right)$.

As noted earlier $\left[\Phi \mid \Phi^{-1}\left(T_{1}\right), \partial\right]=\left[\Phi \mid D_{\omega^{N}}, \partial\right] \neq 0$, which gives the desired contradiction. This completes the proof of the lemma.

\section{A lower estimate on the intersection numbers}

In this section, we apply Lemma 7.2 to obtain the estimate (5.1).

\subsection{PL-approximation}

Fix a parameter $j \in J$; write $D$ for the simplex $D_{j}$ used for slicing, and

$$
F=f^{-1} \mid D \text {. }
$$

Let $\Pi:\left(\mathbf{R}^{3} / W h\right) \times \mathbf{R}^{m} \rightarrow \mathbf{R}^{3} / W h$ be the projection map

$$
\Pi(x, z)=x .
$$

The goal of this subsection is to construct a single-valued version of $\pi^{-1} \Pi F \mid D: D \rightarrow$ $\mathbf{R}^{3}$ whose image intersects the boundaries of the tori transversally.

For the purpose of applying a PL-approximation (Theorem 9.5 in the Appendix), the manifold $\left(\mathbf{R}^{3} / W h\right) \times \mathbf{R}^{m}$ is equipped with a PL-structure and a metric, induced from $\mathbf{R}^{3+m}$ by a fixed homeomorphism (unrelated to $\left.f\right) h:\left(\mathbf{R}^{3} / W h\right) \times \mathbf{R}^{m} \rightarrow \mathbf{R}^{3+m}$, 
which is $\pi^{-1} \times$ id outside $\widetilde{T}_{K+1} \times \mathbf{R}^{m}$ and is diffeomorphic off $\mathcal{L}$. Fix a large integer $K>k_{0}$ and let

$$
\epsilon=c_{0} a^{K+2} / 30 .
$$

From Theorem 9.5 it follows that for any $\epsilon^{\prime}>0, F \mid D$ can be $\epsilon^{\prime}$-approximated by a locally flat PL embedding $L: D \hookrightarrow\left(\mathbf{R}^{3} / W h\right) \times \mathbf{R}^{m}$. When $\epsilon^{\prime}$ is sufficiently small, the approximation satisfies

$$
d_{\delta_{a}}(F(x), L(x))<\epsilon, \quad \text { for all } x \in D \backslash f\left(\widetilde{T}_{K+1} \times \mathbf{R}^{m}\right),
$$

with respect to the original metric. Adjusting $L$ to one in general position with respect to the boundaries of the tori, we may assume that $L(D)$ and $\left(\partial \widetilde{t}_{k_{0}} \cup \bigcup_{k=k_{0}}^{K} \partial \widetilde{T}_{k+1}\right) \times \mathbf{R}^{m}$ meet transversally and their intersection is an 1-manifold having no boundary. Adjusting $L$ again, we may further assume that the projection $\Pi$ embeds each simplex in $L(D) \backslash\left(\widetilde{T}_{K+1} \times \mathbf{R}^{m}\right)$, that $\Pi L(D)$ and $\partial \widetilde{t}_{k_{0}} \cup \bigcup_{k=k_{0}}^{K} \partial \widetilde{T}_{k+1}$ meet transversally and that $(\Pi L)^{-1}\left(\partial \tilde{t}_{k_{0}} \cup \bigcup_{k=k_{0}}^{K} \partial \widetilde{T}_{k+1}\right)$ is a 1 -manifold in $D$ having no boundary. See Rourke and Sanderson [27, Chapter 5], Rushing [28, page 33] and Theorem 9.6 in the Appendix.

Note from (6.2), (6.6), (8.2) and (8.3) that $L(\partial D)$ is homotopic to the meridian $\alpha$ of $\tilde{T}_{k_{0}} \times\{0\}$ in $\tilde{V}_{k_{0}} \times \mathbf{R}^{m}$. By Proposition 2.1,

$$
L(\partial D)=\partial L(D) \text { is not contractible in }\left(\widetilde{T}_{0} \times \mathbf{R}^{m}\right) \backslash \mathcal{L} ;
$$

and by (6.7), (8.2) and (8.3),

(8.5) $q \in \Pi L(D), L(D) \cap \mathcal{L} \neq \varnothing \quad$ and $\quad L(D) \subset\left(\widetilde{T}_{1} \cup \tilde{V}_{1}\right) \times\left[-C_{2}-1, C_{2}+1\right]^{m}$.

The function $\pi^{-1} \Pi L$ is not single-valued on the set $L^{-1}(\mathcal{L}) \cap D$; we modify $\pi^{-1} \Pi L$ near $L^{-1}(\mathcal{L})$ as follows. Let $\Lambda_{1}, \Lambda_{2}, \ldots, \Lambda_{N}$ be the 2 -cells in $D$ enclosed by the outermost components of $L^{-1}\left(\partial\left(\widetilde{T}_{K} \times \mathbf{R}^{m}\right)\right)$. Then the 2 -cells $\Lambda_{1}, \Lambda_{2}, \ldots, \Lambda_{N}$ are mutually disjoint,

$$
L^{-1}(\mathcal{L}) \subset \bigcup_{1}^{N} \Lambda_{n}, \quad \text { and } L\left(D \backslash \bigcup_{1}^{N} \Lambda_{n}\right) \cap\left(\widetilde{T}_{K} \times \mathbf{R}^{m}\right)=\varnothing .
$$

Note however $L\left(\bigcup_{1}^{N} \Lambda_{n}\right)$ need not be contained in $\widetilde{T}_{K} \times \mathbf{R}^{m}$. Since the homeomorphism $h:\left(\mathbf{R}^{3} / W h\right) \times \mathbf{R}^{m} \rightarrow \mathbf{R}^{3+m}$ providing the PL-structure on $\left(\mathbf{R}^{3} / W h\right) \times \mathbf{R}^{m}$ is $\pi^{-1} \times$ id outside $\widetilde{T}_{K+1} \times \mathbf{R}^{m}$, the function $\Phi: D \rightarrow \mathbf{R}^{3}$ defined by

$$
\Phi= \begin{cases}\pi^{-1} \Pi L & \text { in } D \backslash \bigcup_{1}^{N} \Lambda_{n}, \\ \Pi^{\prime} h L & \text { in } \bigcup_{1}^{N} \Lambda_{n},\end{cases}
$$


is continuous on $D$. Here $\Pi^{\prime}: \mathbf{R}^{3} \times \mathbf{R}^{m} \rightarrow \mathbf{R}^{3}$ is the projection with $\Pi^{\prime}(x, z)=x$. From the discussion leading to (8.4) and (8.5), it follows that

$$
\begin{aligned}
& \Phi(\partial D)=\pi^{-1} \Pi L(\partial D) \subset V_{k_{0}} \text { and } \Phi(D) \subset T_{1} \cup V_{1} \subset T_{0}, \\
& \Phi(\partial D) \text { is not contractible in } T_{0} \backslash W h, \\
& \Phi(D) \cap t_{k_{0}} \neq \varnothing \text { and } \Phi(D) \cap T_{k} \neq \varnothing \text { for all } k \geq k_{0}+1 .
\end{aligned}
$$

Define also a single-valued version of $\pi^{-1} \Pi F$ :

$$
\Psi= \begin{cases}\pi^{-1} \Pi F & \text { in } D \backslash \bigcup_{1}^{N} \Lambda_{n}, \\ \Pi^{\prime} h F & \text { in } \bigcup_{1}^{N} \Lambda_{n} .\end{cases}
$$

The $\epsilon$-closeness (8.3) of $F$ and $L$ implies that $\Psi \mid\left(D \backslash \bigcup_{1}^{N} \Lambda_{n}\right)$ and $\Phi \mid\left(D \backslash \bigcup_{1}^{N} \Lambda_{n}\right)$ are $\epsilon$-close with respect to the metric $d_{a}$ on $\mathbf{R}^{3} \backslash W h$.

\subsection{Intersection}

We now apply Lemma 7.2 to torus pairs $\left(t_{k_{0}}, T_{k_{0}+1}\right),\left(T_{k_{0}+1}, T_{k_{0}+2}\right), \ldots,\left(T_{K-1}, T_{K}\right)$. Notice that given any such pair, there is a homeomorphism of $\mathbf{S}^{3}$ that maps the first torus in the pair onto $T_{0}$ and the second one onto $T_{1}$.

To begin, let $\omega_{k_{0}}$ be an innermost component of $\Phi^{-1}\left(t_{k_{0}}\right)$ on which $\Phi$ is interioressential. By (8.6), $\omega_{k_{0}}$ must exist and is nonempty. Following the argument in Lemma 7.2 for the statement that $\Phi \mid \omega^{1}$ is virtually interior-essential, we may conclude that $\Phi:\left(\omega_{k_{0}}, \partial\right) \rightarrow\left(t_{k_{0}}, \partial\right)$ is virtually interior-essential; here the fact that neither a meridian of $t_{k_{0}}$ nor a longitude of $t_{k_{0}}$ is contractible in $T_{0} \backslash \operatorname{Int}\left(t_{k_{0}}\right)$ is needed.

Applying Lemma 7.2 to the torus pair $\left(t_{k_{0}}, T_{k_{0}+1}\right)$, we conclude $\Phi^{-1}\left(T_{k_{0}+1}\right)$ contains at least two components $\omega_{k_{0}+1}^{1}$ and $\omega_{k_{0}+1}^{2}$ on which the maps $\Phi \mid \omega_{k_{0}+1}^{i}: \omega_{k_{0}+1}^{i} \rightarrow$ $T_{k_{0}+1}(i=1,2)$ are virtually interior-essential. Apply Lemma 7.2 again to each $\omega_{k_{0}+1}^{i}(i=1,2)$ to obtain at least four components $\omega_{k_{0}+2}^{i}(1 \leq i \leq 4)$ of $\Phi^{-1}\left(T_{k_{0}+2}\right)$ on which the maps $\Phi \mid \omega_{k_{0}+2}^{i}: \omega_{k_{0}+2}^{i} \rightarrow T_{k_{0}+2}(1 \leq i \leq 4)$ are virtually interioressential. Continuing inductively, we get for each $k, k_{0}+1 \leq k \leq K-1$, at least $2^{k-k_{0}}$ components $\omega_{k}^{i}\left(1 \leq i \leq 2^{k-k_{0}}\right)$ of $\Phi^{-1}\left(T_{k}\right)$ on which $\Phi$ is virtually interioressential. In fact, the proof of Lemma 7.2 also shows that for a fixed $k$, the minimal 2-cells $D_{\omega_{k}^{i}}$ in $D$ containing $\omega_{k}^{i}\left(1 \leq i \leq 2^{k-k_{0}}\right)$ are mutually disjoint, and that each $\Phi\left(\partial D_{\omega_{k}^{i}}\right)$ is a nonzero multiple of a meridian of $T_{k}$.

Fix an $\omega_{k}^{i}$ with $k_{0}+1 \leq k \leq K-1$ and $1 \leq i \leq 2^{k-k_{0}}$. For any $\gamma$ in the curve family $\mathcal{C}_{k}$ defined in Section $4, \pi^{-1} \gamma$ (a core of $T_{k}$ ) and $\Phi\left(\partial D_{\omega_{k}^{i}}\right.$ ) are linked. So $\pi^{-1} \gamma$ intersects $\Phi\left(D_{\omega_{k}^{i}}\right)$, in fact intersects $\Phi\left(\omega_{k}^{i}\right)$. 
For each $\gamma \in \mathcal{C}_{k}$, the core $\pi^{-1} \gamma$ intersects $\Psi\left(\omega_{k}^{i}\right)$ also. This can be seen as follows. Since $\pi^{-1} \gamma \subset U_{k} \subset t_{k}$ and $\Phi\left(\partial D_{\omega_{k}^{i}}\right)=\pi^{-1} \Pi L\left(\partial D_{\omega_{k}^{i}}\right) \subset \partial T_{k}$, we have

$$
30 \epsilon=c_{0} a^{K+2} \leq \operatorname{dist}_{d_{a}}\left(t_{k}, \partial T_{k}\right) \leq \operatorname{dist}_{d_{a}}\left(\pi^{-1} \gamma, \Phi\left(\partial D_{\omega_{k}^{i}}\right)\right) .
$$

From the $\epsilon$-closeness of $\Psi$ and $\Phi$, it follows that $\Psi\left(\omega_{k}^{i}\right)$ is contained in $V_{k} \cup T_{k}$, a neighborhood of $T_{k}$, and that $\Psi \mid \partial \omega_{k}^{i}$ and $\Phi \mid \partial \omega_{k}^{i}$ are homotopic in $V_{k} \backslash U_{k}$, a neighborhood of $\partial T_{k}$ outside $t_{k}$. So $\pi^{-1} \gamma$ and $\Psi\left(\partial D_{\omega_{k}^{i}}\right)$ are also linked. Therefore $\pi^{-1} \gamma$ intersects $\Psi\left(D_{\omega_{k}^{i}}\right)$, in fact intersects $\Psi\left(\omega_{k}^{i}\right)$.

The intersection of $\pi^{-1} \gamma$ and $\Psi\left(\omega_{k}^{i}\right)$ necessarily takes place at points in $U_{k}$, which is outside the torus $T_{K}$. Therefore $\Psi=\pi^{-1} \Pi F$ at the points of intersection. So $\gamma$ intersects $\Pi F\left(\omega_{k}^{i}\right)$ for each $\gamma \in \mathcal{C}_{k}$. Consequently, $\Gamma$ intersects $F\left(\omega_{k}^{i}\right)$ for each $\Gamma \in \mathcal{S}_{k}$.

This together with Remark 6.5 shows that for each $k, k_{0}+1 \leq k \leq K-1$,

$$
N(k, \Gamma, j) \geq 2^{k-k_{0}} \quad \text { for all } \Gamma \in \mathcal{S}_{k} \text { and } j \in J .
$$

Since $K$ can be arbitrarily large, estimate (5.1) follows. This completes the proof of Theorem 1.1.

\section{Appendix}

We state some topological facts needed in our proof.

The first theorem is about the size of the complement of a non-simply connected set proved by Martio, Rickman and Väisälä [23].

9.1 Theorem Suppose that $G$ is a simply connected domain in $\mathbf{R}^{n}(n \geq 3)$ and $A$ is a closed subset of $G$. If $G \backslash A$ is not simply connected, then $A$ must have positive (n-2)-dimensional Hausdorff measure.

Next is a theorem about PL-approximation of embeddings of one-dimensional complexes due to Moise [25, page 46].

9.2 Theorem Let $K^{1}$ be a 1-dimensional complex, $f:\left|K^{1}\right| \hookrightarrow \mathbf{R}^{2}$ an embedding, and $\psi>0$ a continuous function on $\left|K^{1}\right|$. Then there exists a piecewise linear embedding $\theta:\left|K^{1}\right| \hookrightarrow \mathbf{R}^{2}$ such that

(1) $|\theta(x)-f(x)|<\psi(x)$ for all $x \in\left|K^{1}\right|(\psi$-approximation of $f)$,

(2) $\theta(v)=f(v)$ for all vertices $v$ of $K^{1}$. 
9.3 Remark The theorem remains true if the target space is $\mathbf{R}^{n}$ for any $n \geq 2$. The proof is essentially unchanged.

The following theorem on unknotting spheres is due to Zeeman [40]; the second sentence in the statement below can be deduced from the proof of Theorem 5.6 in Rourke and Sanderson [27] and the propositions mentioned therein.

9.4 Theorem Any piecewise linear topological 1-sphere $l \subset \mathbf{S}^{n}$ is unknotted in $\mathbf{S}^{n}$ when $n \geq 4$. More precisely, there is a global PL-homeomorphism $L$ of $\mathbf{S}^{n}$ with the following properties:

(1) $L$ maps $l$ onto the boundary of a standard 2-simplex, in $B(Y, 2 \operatorname{diam} l)$ for some point $Y \in l$.

(2) $L \equiv$ id in $\mathbf{S}^{n} \backslash B(Y, 2 \operatorname{diam} l)$.

The following PL-approximation of topological embeddings of $k$-cells into a manifold $M^{n}$ is due to Ancel and Cannon [2] when codimension is 1, due to Miller [24] and Černavskiı̌ [8] when codimension is 3 or higher and due to Venema [37; 38] when codimension is 2 . We need it for $k=2$ and $n \geq 4$.

9.5 Theorem Let $h: I^{k} \rightarrow M^{n}$ be a topological embedding of the closed $k$-cell $I^{k}$ into a piecewise linear $n-$ manifold $M^{n}$ and $n-k \geq 1$. Then for every $\epsilon>0$ there exists a locally flat piecewise linear embedding $L: I^{k} \rightarrow M^{n}$ such that $d(h(x), L(x)) \leq \epsilon$ for each $x \in I^{k}$.

The following theorem on transversality is well-known (see for example Daverman [9, page 69]). When the maps are piecewise linear the proof is simpler.

9.6 Theorem Suppose $M^{n}$ is an $n$-manifold, $\Sigma$ is an $(n-1)$-manifold embedded in $M$ as a closed and bicollared subset, $F: B^{2} \rightarrow M$ is a continuous map, $U$ is an open subset of $M$ containing $\Sigma$, and $\epsilon>0$. Then there exists a map $L: B^{2} \rightarrow M$ satisfying

(1) $L\left|\left(B^{2}-F^{-1} U\right)=F\right|\left(B^{2}-F^{-1} U\right)$,

(2) $d(L, F)<\epsilon$,

(3) $L^{-1} \Sigma$ is a 1-manifold in $B^{2}$ with $\partial\left(L^{-1} \Sigma\right)=\partial B^{2} \cap L^{-1} \Sigma$,

(4) for any point $x \in L^{-1} \Sigma, L\left(B^{2}\right)$ pierces $\Sigma$ at $L(x)$, ie, for each neighborhood $W$ of $x$ in $B^{2}, L(W)$ touches both sides of the bicollar on $\Sigma$ near $L(x)$.

We say $L\left(B^{2}\right)$ and $\Sigma$ meet transversally if the map $L: B^{2} \rightarrow M$ satisfies (3) and (4). 


\section{References}

[1] L Ahlfors, A Beurling, Conformal invariants and function-theoretic null-sets, Acta Math. 83 (1950) 101-129 MR0036841

[2] F D Ancel, J W Cannon, The locally flat approximation of cell-like embedding relations, Ann. of Math. (2) 109 (1979) 61-86 MR519353

[3] J J Andrews, L Rubin, Some spaces whose product with $E^{1}$ is $E^{4}$, Bull. Amer. Math. Soc. 71 (1965) 675-677 MR0176454

[4] $\mathbf{R}$ H Bing, The cartesian product of a certain nonmanifold and a line is $E^{4}$, Ann. of Math. (2) 70 (1959) 399-412 MR0107228

[5] $\mathbf{R} \mathbf{H}$ Bing, A set is a 3 cell if its cartesian product with an arc is a 4 cell, Proc. Amer. Math. Soc. 12 (1961) 13-19 MR0123303

[6] M Bonk, B Kleiner, Quasisymmetric parametrizations of two-dimensional metric spheres, Invent. Math. 150 (2002) 127-183 MR1930885

[7] J W Cannon, The recognition problem: what is a topological manifold?, Bull. Amer. Math. Soc. 84 (1978) 832-866 MR0494113

[8] A V Černavskiü, Piece-wise linear approximation of embeddings of manifolds in codimensions greater than two. A supplement to the article "Piece-wise linear approximation of embeddings of cells and spheres in codimensions greater than two", Mat. Sb. (N.S.) 82 (124) (1970) 499-500 MR0275438

[9] R J Daverman, Decompositions of manifolds, Pure and Applied Math. 124, Academic Press, Orlando, FL (1986) MR872468

[10] G David, S Semmes, Quantitative rectifiability and Lipschitz mappings, Trans. Amer. Math. Soc. 337 (1993) 855-889 MR1132876

[11] R D Edwards, Suspensions of homology spheres arXiv:math/0610573

[12] R D Edwards, The topology of manifolds and cell-like maps, from: "Proceedings of the International Congress of Mathematicians (Helsinki, 1978)", Acad. Sci. Fennica, Helsinki (1980) 111-127 MR562601

[13] H Federer, Geometric measure theory, Grund. der math. Wissenschaften 153, Springer, New York (1969) MR0257325

[14] M H Freedman, R Skora, Strange actions of groups on spheres, J. Differential Geom. 25 (1987) 75-98 MR873456

[15] B Fuglede, Extremal length and functional completion, Acta Math. 98 (1957) 171-219 MR0097720

[16] F W Gehring, Rings and quasiconformal mappings in space, Trans. Amer. Math. Soc. 103 (1962) 353-393 MR0139735

[17] F W Gehring, The $L^{p}$-integrability of the partial derivatives of a quasiconformal mapping, Acta Math. 130 (1973) 265-277 MR0402038 
[18] J Heinonen, P Koskela, Definitions of quasiconformality, Invent. Math. 120 (1995) 61-79 MR1323982

[19] J Heinonen, P Koskela, Quasiconformal maps in metric spaces with controlled geometry, Acta Math. 181 (1998) 1-61 MR1654771

[20] J Heinonen, S Semmes, Thirty-three yes or no questions about mappings, measures, and metrics, Conform. Geom. Dyn. 1 (1997) 1-12 MR1452413

[21] R C Kirby, The topology of 4-manifolds, Lecture Notes in Math. 1374, Springer, Berlin (1989) MR1001966

[22] T J Laakso, Plane with $A_{\infty}$-weighted metric not bi-Lipschitz embeddable to $\mathbb{R}^{N}$, Bull. London Math. Soc. 34 (2002) 667-676 MR1924353

[23] O Martio, S Rickman, J Väisälä, Topological and metric properties of quasiregular mappings, Ann. Acad. Sci. Fenn. Ser. A I (1971) 31 MR0299782

[24] R T Miller, Approximating codimension 3 embeddings, Ann. of Math. (2) 95 (1972) 406-416 MR0307246

[25] E E Moise, Geometric topology in dimensions 2 and 3, Graduate Texts in Math. 47, Springer, New York (1977) MR0488059

[26] S Rickman, Quasiregular mappings, Ergebnisse der Math. und ihrer Grenzgebiete (3) 26, Springer, Berlin (1993) MR1238941

[27] C P Rourke, B J Sanderson, Introduction to piecewise-linear topology, Springer Study Edition (1982) MR665919

[28] T B Rushing, Topological embeddings, Pure and Applied Math. 52, Academic Press, New York (1973) MR0348752

[29] S Semmes, Chord-arc surfaces with small constant. II. Good parameterizations, Adv. Math. 88 (1991) 170-199 MR1120612

[30] S Semmes, Finding curves on general spaces through quantitative topology, with applications to Sobolev and Poincaré inequalities, Selecta Math. (N.S.) 2 (1996) 155-295 MR1414889

[31] S Semmes, Good metric spaces without good parameterizations, Rev. Mat. Iberoamericana 12 (1996) 187-275 MR1387590

[32] S Semmes, On the nonexistence of bi-Lipschitz parameterizations and geometric problems about $A_{\infty}$-weights, Rev. Mat. Iberoamericana 12 (1996) 337-410 MR1402671

[33] S Semmes, Some topics concerning homeomorphic parameterizations, Publ. Mat. 45 (2001) 3-67 MR1829576

[34] L Siebenmann, D Sullivan, On complexes that are Lipschitz manifolds, from: "Geometric topology (Proc. Georgia Topology Conf., Athens, Ga., 1977)”, (J C Cantrell, editor), Academic Press, New York (1979) 503-525 MR537747 
[35] P Tukia, J Väisälä, Quasisymmetric embeddings of metric spaces, Ann. Acad. Sci. Fenn. Ser. A I Math. 5 (1980) 97-114 MR595180

[36] J Väisälä, Lectures on n-dimensional quasiconformal mappings, Lecture Notes in Math. 229, Springer, Berlin (1971) MR0454009

[37] G A Venema, Approximating disks in 4-space, Michigan Math. J. 25 (1978) 19-27 MR497879

[38] G A Venema, Approximating codimension two embeddings of cells, Pacific J. Math. 126 (1987) 165-195 MR868611

[39] J HC Whitehead, A certain open manifold whose group is unity, Quart. J. Math. Oxford (2) 6 (1935) 268-279

[40] E C Zeeman, Unknotting spheres, Ann. of Math. (2) 72 (1960) 350-361 MR0117738

Department of Mathematics, University of Michigan 530 Church Street, Ann Arbor, MI 48109-1043, USA

Department of Mathematics, University of Illinois 1409 West Green Street, Urbana, IL 61822, USA

wu@math.uiuc.edu

Proposed: Colin Rourke

Seconded: Tobias Colding, David Gabai
Received: 5 March 2008

Revised: 28 October 2009 